\title{
Effet de l'implantation des souches de Panicum maximum var. C1 sur l'invasion de Hyptis suaveolens (L.) Poit dans les pâturages naturels au Nord-Bénin
}

\author{
Souhaibou YACOUBOU IBRAHIMA ${ }^{1}$, Alain S. YAOITCHA ${ }^{1,2^{*}}$, Paolo LESSE ${ }^{1}$, \\ André B. $\mathrm{ABOH}^{3}$ et Marcel R. B. HOUINATO ${ }^{1}$ \\ ${ }^{1}$ Laboratoire d'Ecologie Appliquée/Faculté des Sciences Agronomiques/Université d'Abomey-Calavi, 01 BP \\ 526, Cotonou, Bénin. \\ ${ }_{2}^{2}$ Institut National des Recherches Agricoles du Bénin, 01 BP 884 Cotonou, Bénin. \\ ${ }^{3}$ Université Nationale d'Agriculture, Bénin. \\ *Auteur correspondant ; E-mail : ayaoitcha@gmail.com; Tel. +222997778444
}

\author{
Received: 13-10-2020 \\ Accepted: 26-02-2021 \\ Published: 28-02-2021
}

\section{RESUME}

La productivité et la durabilité des pâturages naturels sont menacées au Nord-Bénin par la prolifération de Hyptis suaveolens. La restauration de ces pâturages nécessite des mesures de lutte contre cette plante. L'étude visait à évaluer l'effet de Panicum maximum $\mathrm{C} 1$ installé avec des souches à une densité de 4 plants $/ \mathrm{m}^{2}$ sur $H$. suaveolens et à comparer les réactions face à $H$. suaveolens des souches de $P$. maximum issues d'un milieu infesté ou non par $H$. suaveolens. Le contrôle d'invasion de $H$. suaveolens par la culture de $P$. maximum a été évalué par la contribution spécifique de contact (CSC), le biovolume $(\mathrm{Bv})$ et la biomasse $(\mathrm{Bm})$ de H. suaveolens. Les résultats ont montré que la présence de $P$. maximum $\mathrm{C} 1$ a réduit significativement $(\mathrm{p}<0,05)$ la $\mathrm{CSC}$, le $\mathrm{Bv}$ et la $\mathrm{Bm}$ de $H$. suaveolens respectivement à $13,20 \%, 0,16 \%$ et $15,62 \%$ après trois mois de culture sans exploitation. La provenance des souches n'a eu d'effet significatif $(\mathrm{p}>0,05)$ sur aucun des paramètres biophysiques de $H$. suaveolens, ni sur le développement de $P$. maximum $\mathrm{C} 1$ en présence de $H$. suaveolens. Ainsi, la culture de $P$. maximum dans les pâturages envahis a permis d'améliorer leurs caractéristiques pastorales et peut être promue en milieu paysan.

(C) 2021 International Formulae Group. All rights reserved.

Mots clé : Hyptis suaveolens, Panicum maximum C1, plante envahissante, pâturages, Bénin.

\section{Effect of Panicum maximum var. C1 stumps cultivation on invasion of Hyptis suaveolens $(\mathrm{L}$.$) Poit in natural pastures in northern Benin$}

\begin{abstract}
The productivity and sustainability of natural pastures in northern Benin are threatened by the proliferation of Hyptis suaveolens. The restoration of these pastures requires control measures against this plant. This study aimed at evaluating the effect of Panicum maximum $\mathrm{C} 1$ installed with stumps at a density of 4 plants $/ \mathrm{m}^{2}$ on the development of $H$. suaveolens and to compare the reactions with respect to $H$. suaveolens of $P$. maximum's stumps from an infested environment or not by $H$. suaveolens. Invasion control of $H$. suaveolens by culture of $P$.
\end{abstract}


maximum was assessed using the specific contact contribution (SCC), biovolume (Bv) and biomass (Bm) of $H$. suaveolens. The results showed that the presence of $P$. maximum $\mathrm{C} 1$ reduced significantly $(\mathrm{p}<0.05)$ the $\mathrm{SCC}$, $\mathrm{Bv}$ and $\mathrm{Bm}$ of $H$. suaveolens respectively to $13.20 \%, 0.16 \%$ and $15.62 \%$ after three months of cultivation without exploitation. The origin of stumps had no significant effect ( $\mathrm{p}>0.05$ ) on any of the biophysical parameters of $H$. suaveolens, nor on the development of $P$. maximum $\mathrm{C} 1$ in the presence of $H$. suaveolens. Thus, the cultivation of $P$. maximum in pastures invaded has improved their pastoral characteristics and can be promoted in rural areas. (C) 2021 International Formulae Group. All rights reserved.

Keywords: Hyptis suaveolens, Panicum maximum C1, invasive plant, pastures, Benin.

\section{INTRODUCTION}

La prolifération des espèces indésirables envahissantes au détriment des bonnes graminées appétées par les herbivores constitue une contrainte majeure pour le développement de l'élevage en Afrique au Sud du Sahel. En effet, les espèces envahissantes, particulièrement agressives, colonisent les pâturages par étouffement progressif des espèces fourragères locales (Aboh et al., 2012a) réduisant ainsi la productivité de ces pâturages. Parmi les espèces envahissantes, Hyptis suaveolens Poit. (Lamiaceae) s'est révélée la plus envahissante compte tenu de son cycle biologique (Thiombiano et al., 2009). Son impact négatif sur la valeur quantitative et qualitative des pâturages naturels a été signalé par plusieurs auteurs (Thiombiano, 2008; Aboh et al., 2009; Oumorou et al., 2010 ; Aboh et al., 2012a).

Au Bénin, plus particulièrement dans la partie septentrionale du pays, $H$. suaveolens envahit les bords de route, les champs, les jachères, les friches et surtout les pâturages naturels. Les perturbations engendrées par cette plante envahissante au niveau des parcours naturels sont à l'origine des difficultés qu'éprouvent les éleveurs pour nourrir les ruminants.

Face à ces difficultés, les éleveurs développent des stratégies d'adaptation (Oumorou et al., 2010; Aboh et al., 2012a, 2012b) sans suite favorable pour l'activité d'élevage. Les méthodes telles que l'arrachage, le sarclage et les feux de végétation ont montré leurs limites d'efficacité contre cette plante
(CABI, 2004; Holou et al., 2013). Les tentatives de contrôle de la plante à travers la culture des graminées compétitives telles que Panicum maximum $\mathrm{C} 1$ ont donné des résultats intéressants (D’Antonio and Meyerson, 2002). Cependant, les résultats de ces études restent encore à approfondir et à être adaptés au milieu réel avant d'être proposés comme solution aux éleveurs.

En effet, les travaux effectués par Aboh (2008) ont été conduits avec des souches de $P$. maximum $\mathrm{C} 1$ à des densités très fortes telles que : 25 plants $/ \mathrm{m}^{2}$ et 16 plants $/ \mathrm{m}^{2}$. Pour la valorisation des résultats d'une telle recherche bénéfique pour la population, les densités raisonnables doivent être envisagées. En effet, l'installation d'une parcelle de $P$. maximum $\mathrm{C} 1$ à une densité très serrée de repiquage de souche est très contraignante surtout quand il s'agit d'emblaver de grandes superficies. Il est ainsi important d'essayer une densité plus réduite et adaptée au milieu réel telle: 4 plants $/ \mathrm{m}^{2}$ $(0,80 \mathrm{~m} \times 0,80 \mathrm{~m})$ et d'évaluer son effet sur les caractéristiques biophysiques de $H$. suaveolens.

Par ailleurs, d'autres études réalisées ces dernières années pour le contrôle de l'invasion ont montré que les plantes d'une espèce compétitive ayant survécu dans un milieu infesté par une plante envahissante sont plus performantes que celles issus d'un milieu non infesté (Ferrero-Serrano et al., 2011 ; Sebade et al., 2012). Dans le cas de $P$. maximum $\mathrm{C} 1$ qui est une espèce caractérisée par une grande capacité étouffante des espèces en présence (César, 2005), aucune étude de 
compétitivité n'est encore réalisée. Ainsi, la question fondamentale qui se pose est de savoir s'il y a une différence de réponse entre les souches de $P$. maximum $\mathrm{C} 1$ issues d'un milieu infesté par $H$. suaveolens et celles provenant d'un milieu non infesté.

Ainsi, l'objectif de cette étude était (i) d'évaluer l'effet de l'introduction de P. maximum $\mathrm{C} 1$ sur $H$. suaveolens et les autres espèces et (ii) de comparer les performances des souches de $P$. maximum $\mathrm{C} 1$ provenant d'un milieu infesté par $H$. suaveolens avec celles provenant d'un milieu non infesté.

\section{MATERIEL ET METHODES}

\section{Milieu d'étude}

L'étude a été réalisée sur la station de recherche du Centre de Recherches Agricoles Nord-Est basé à Ina dans la Commune de Bembéréké (E 2०44'20" et $\mathrm{N}^{\circ} 9^{\circ} 58^{\prime} 32^{\prime \prime}$ " (Figure 1). La zone Nord constitue un lieu privilégié où se confrontent toutes les logiques du développement de l'élevage transhumant. Cette partie du pays représente une zone de concentration du cheptel national et de prédilection de transhumants étrangers (Lesse, 2016). Elle constitue une zone d'accueil par excellence des transhumants aussi bien nationaux qu'internationaux en raison de la superficie couverte par les forêts classées qui sont dominées par des savanes arborées soudano-guinéennes (Faure, 1977; Sinsin, 1993). La forte fréquentation de ces zones par les troupeaux autochtones et ceux des étrangers, a certainement influencé la forte invasion des pâturages naturels exploités par $H$. suaveolens.

Le climat de la zone d'étude est de type tropical caractérisé par une saison pluvieuse (mai à octobre) et une saison sèche (novembre à avril). La pluviométrie de l'année 2017, l'année à laquelle l'étude a été réalisée, est de 1322,3 mm, répartie sur 69 jours (CRA Nord, 2018).

La température moyenne annuelle est d'environ $26^{\circ} \mathrm{C}$ et l'humidité relative moyenne de l'air est de 78-96\% au cours de la journée des mois pluvieux. Le sol du site appartient à la classe des sols ferrugineux tropicaux avec des réserves en phosphores assez faibles $(0,1$ à $0,25 \%)$, le pH plus ou moins égal à 6 et les teneurs en matières organiques voisines de 0,4\% (Willaine, 1965 ; Youssouf, 1990).

\section{Dispositif expérimental}

Le dispositif expérimental a été un bloc aléatoire complet (BAC) à trois (3) traitements (T0, TS, TSI) répétés trois fois. Les 3 traitements ci-dessous ont été aléatoirement affectés à chacune des 3 parcelles d'une superficie de $10 \mathrm{~m}$ x $5 \mathrm{~m}$ au niveau d'une répétition.

- T0 : Parcelle Témoin sans ensemencement de $P$. maximum $\mathrm{C} 1$;

- TS : Parcelle ensemencée avec les éclats de souches provenant d'un milieu non infesté

- TSI : Parcelle ensemencée avec les éclats de souches provenant d'un milieu infesté.

Les éclats de souche de 2 à 3 talles et hauts de $20 \mathrm{~cm}$ (Messager, 1984) ont été implantés suivant un écartement de $0,80 \mathrm{~m}$ x $0,80 \mathrm{~m}$. L'essai a été implanté sur une jachère préalablement envahie par $H$. suaveolens. Avant l'installation, le terrain a été sarclé, labouré à plat puis nivelé. Au cours de l'expérimentation, aucun entretien ou sarclage n'a été effectué sur les parcelles excepté les allées. La reprise des éclats de souche de $P$. maximum $\mathrm{C} 1$ a été suivie et les souches mortes ont été régulièrement remplacées.

\section{Collecte des données}

Avant l'implantation de l'essai en juin, un relevé de végétation a été fait pour évaluer l'abondance du pâturage envahi par $H$. suaveolens.

Sur les parcelles de l'essai, les données ont été collectées en utilisant les méthodes que sont : le relevé linéaire de Daget et Poissonet (1971) suivant les diagonales des parcelles de façon croisée a été utilisée pour la collecte des données, les mesures de hauteur et de diamètre 
des plants et la coupe de biomasse.

Les relevés linéaires ont été effectués un mois après l'installation et tous les mois jusqu'à la fin de la saison végétative, soit trois (3) séries de données. La méthode des pointquadras alignés de Daget et Poissonet (1971) suivant les diagonales des parcelles de façon croisée a été utilisée pour la collecte des données. Au total, 100 points contacts ont été parcourus par parcelle.

Pour les mesures de hauteur et de diamètre, cinq (05) placettes permanentes de 1 $\mathrm{m}^{2}$ ont été implantées de façon aléatoire sur chaque parcelle. La hauteur et le diamètre des quatre touffes de $P$. maximum $\mathrm{C} 1$ par $\mathrm{m}^{2}$ et de dix plants de $H$. suaveolens par $\mathrm{m}^{2}$ ont été pris. Les dix plants de $H$. suaveolens ont été choisis au hasard et marqués. Le comptage des plants de $H$. suaveolens a été également réalisé à l'intérieur de chaque placette permanente pour obtenir le nombre total des plants par $\mathrm{m}^{2}$. Ces données ont été collectées tous les mois à partir d'un mois après l'installation jusqu'à la fin de la saison végétative, soit trois séries de données.

Enfin, la coupe de biomasse a été réalisée dans les 5 carrés de $1 \mathrm{~m}$ x $1 \mathrm{~m}$ à la fin de la saison végétative en octobre au niveau de chaque parcelle. La méthode de coupe rase au sécateur de jardinier a été utilisée. La biomasse ainsi récoltée a été triée en trois catégories : $P$. maxinum $\mathrm{C} 1, H$. suaveolens et les autres espèces. Le poids des matières fraîches a été mesuré sur le terrain à l'aide d'un peson. Un échantillon de $200 \mathrm{~g}$ de chaque catégorie a été emballé dans des sachets de productivité puis séché à l'étuve jusqu'à l'obtention du poids constant.

\section{Analyse des données}

Le contrôle d'invasion de $H$. suaveolens par la culture de $P$. maximum $\mathrm{C} 1$ a été évalué en utilisant la contribution spécifique de contact (CSC), le biovolume (Bv) et la biomasse $(\mathrm{Bm})$ des catégories d'espèces telles $P$. maximum $\mathrm{C} 1, H$. suaveolens et autres espèces.

Les données de relevé linéaire ont été utilisées pour calculer la contribution spécifique de contact (CSC) de chaque catégorie d'espèces $(P$. maximum $\mathrm{C} 1, H$. suaveolens et autres espèces) suivant la formule de Daget et Poissonet (1971) :

$\boldsymbol{C S C i}=\frac{1}{n} \sum_{i=1}^{n}\left(\frac{F R i}{\sum_{i=1}^{p} F R i} \times 100\right)$, avec :

Csci $=$ Contribution spécifique de contact d'une catégorie d'espèces i en pourcentage ; FRi $=$ Fréquence relative d'une catégorie d'espèces en pourcentage; $p=3$ (Nombre total de catégories d'espèces enregistrées lors du relevé), $n=$ Nombre total de points par parcelle.

Les données de hauteurs et de diamètres avaient servi à calculer le biovolume $(\mathrm{Bv})$ des touffes de $P$. maximum $\mathrm{C} 1$ et des $H$. suaveolens suivant la formule suivante :

Bvi $\left(m^{3}\right)=\frac{\pi}{4 n} \sum_{i=1}^{n}\left(D_{i}^{2} \times H_{i}\right)$, avec :

$\mathrm{Di}=$ Diamètre d'une catégorie d'espèces i et $\mathrm{Hi}$ = Hauteur d'une catégorie d'espèces $\mathrm{i}, \mathrm{n}=$ Nombre total de Touffes/plants de chaque catégorie par $\mathrm{m}^{2} \mathrm{~d}$ 'une parcelle.

La valeur moyenne de biomasse $(\mathrm{Bm})$ de chaque catégorie d'espèces $(P$. maximum $\mathrm{C} 1, H$. suaveolens et Autres espèces) a été calculée et exprimée en $\mathrm{kg}$ de matière sèche par hectare suivant la formule :

$\boldsymbol{B m}(\boldsymbol{K g} / \boldsymbol{M S} / \mathbf{h a})=\frac{1}{n} \sum_{i=1}^{n} B_{i}$, avec :

$\mathrm{Bi}=$ Biomasse d'une catégorie d'espèces par $\mathrm{m}^{2}$ et $\mathrm{n}=$ Nombre total de carrés coupés par parcelle.

La statistique descriptive et les tests d'analyse de variance (ANOVA) suivi du test de Newman-Keuls ont été utilisés pour déterminer d'éventuelles différences significatives entre les moyennes au seuil de $5 \%$. Le logiciel R V.2.15. a été utilisé pour l'analyse des données. 


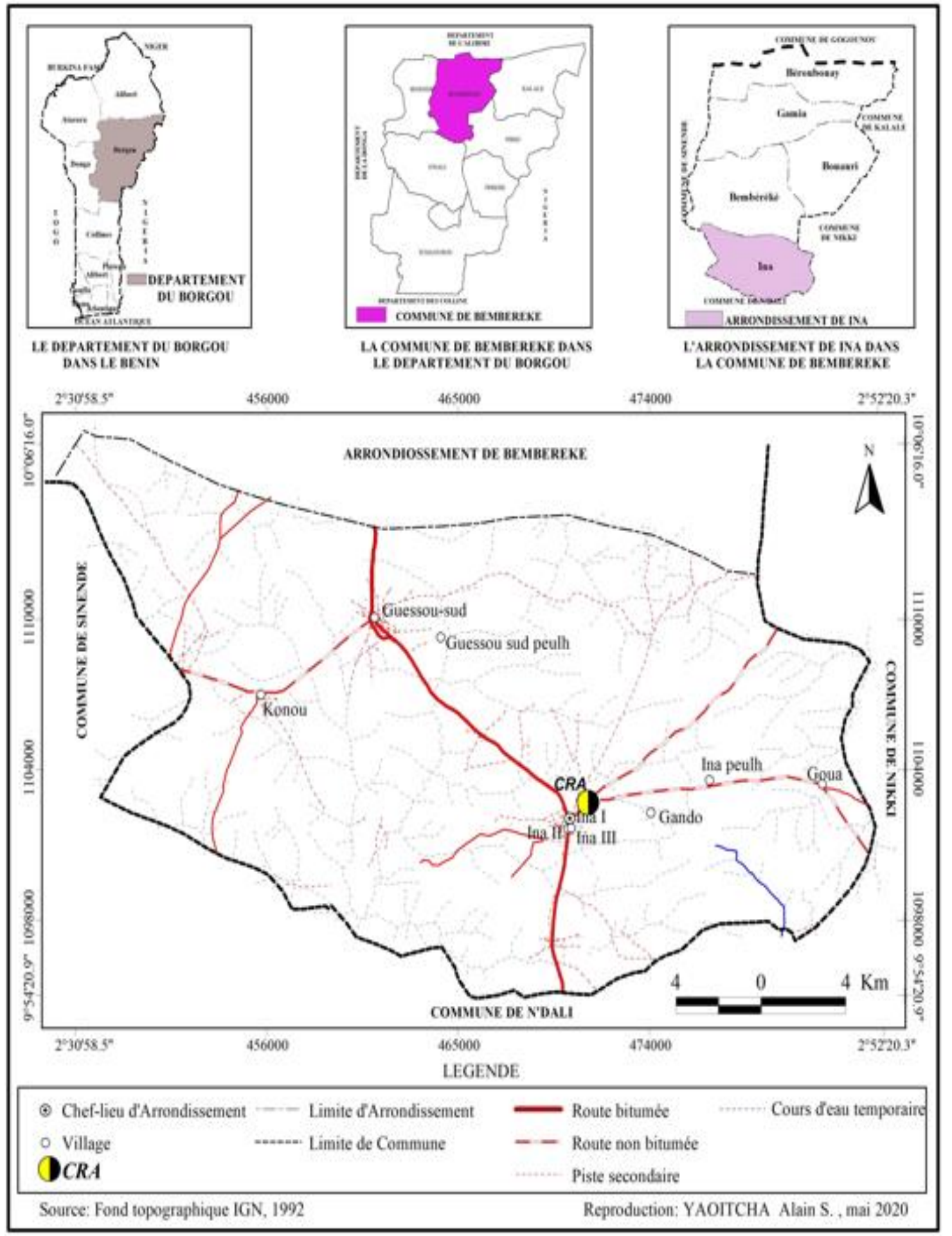

Figure 1 : Localisation de la zone d'étude. 


\section{RESULTATS}

\section{Abondance des espèces du pâturage envahi par H. suaveolens}

Au total, 18 espèces réparties dans 09 familles ont été recensées dans le pâturage envahi par $H$. suaveolens. Les familles les plus fréquentes ont été : les Lamiaceae (47\%), Passifloraceae (23\%) et Fabaceae (19\%) (Figure 2). Les espèces abondantes ont été : $H$. suaveolens (47\%), Passiflora foetida (23\%) et Indigofera hirsuta (16\%). Les autres espèces ont été très faiblement représentées. L'invasion de $H$. suaveolens dans ce pâturage a été exprimée par la forte proportion de Lamiaceae. Les Poaceae et les Cyperaceae qui regroupent les graminées, recherchées par le bétail, ont été très peu représentées $(10,66 \%)$. Il en est de même pour les Fabaceae qui représentent les légumineuses.

\section{Effet de l'introduction de P. maximum C1 sur $\boldsymbol{H}$. suaveolens et les autres espèces}

En général, l'introduction de $P$. maximum $\mathrm{C} 1$ dans le pâturage naturel a réduit significativement (Tableau 1) la fréquence de contact de $H$. suaveolens de $100 \%$ à $13 \%$ après trois mois de culture sans exploitation (Figure 3). En effet, la Contribution Spécifique de Contact (CSC) de H. suaveolens qui, en absence de $P$. maximum $\mathrm{C} 1$, était en moyenne de $26 \%$ sur toute la période de l'essai, s'était amenuit progressivement en présence de $P$. maximum $\mathrm{C} 1$ en passant de $13 \%$ dès le premier mois à $4 \%$ à la fin du troisième mois. $P$. maximum $\mathrm{C} 1$ s'était ainsi recouvré de l'espace révoqué à $H$. suaveolens.

Quant aux autres espèces, leur CSC était importante (74\% en moyenne) mais très peu influencée par la présence de $P$. maximum $\mathrm{C} 1$. Ainsi, à la fin du troisième mois, la CSC des autres espèces était de $72 \%$ en absence de $P$. maximum $\mathrm{C} 1$ contre $68 \%$ en présence de $P$. maximum.

De façon globale, ces autres espèces ont été composées majoritairement des espèces des familles des Cyperaceae (Cyperus esculentus, Cyperus rotondus, Kyllinga erecta, Mariscus alternifolius), des Fabaceae (Alysicarpus ovalifolius, Indigofera hirsuta), des Rubiaceae (Mitracarpus hirtus, Oldenlandia sp), des
Passifloraceae (Passifora $s p$ ) et des poacées (Digitaria horizontalis, Paspalum orbiculare, Paspalum polystachyum, Setaria pallidefusca).

En termes de biovolume, celui de $H$. suaveolens était réduit significativement (Tableau 1) de $0,11 \mathrm{~m}^{3} / \mathrm{m}^{2}$ en absence $P$. maximum $\mathrm{C} 1$ à $0,0265 \mathrm{~m}^{3} / \mathrm{m}^{2}$ en présence de $P$. maximum $\mathrm{C} 1$, dès le premier mois après l'installation (Tableau 1). A la fin du troisième mois, le biovolume de $H$. suaveolens s'était réduit à $0,008 \mathrm{~m}^{3} / \mathrm{m}^{2}$ tandis que celui de $P$. maximum $\mathrm{C} 1$ s'était accrut à $4,95 \mathrm{~m}^{3} / \mathrm{m}^{2}$. Ainsi, $H$. suaveolens n'occupait que $0,001 \%$ du volume occupé par les végétaux par l'unité de $\mathrm{m}^{2}$.

Après trois mois, la culture de $P$. maximum $C 1$ a réduit significativement (Tableau 1) la biomasse de H. suaveolens de $2027 \mathrm{Kg} / \mathrm{ha}$ à $375 \mathrm{Kg} / \mathrm{ha}$ (soit 84,38\%). La biomasse de $H$. suaveolens révoquée a été ainsi reconquise en $P$. maximum $C l$ qui constitue une bonne pâture pour les animaux (Tableau $1)$.

\section{Performance des souches de $P$. maximum $\mathrm{C} 1$ provenues d'un milieu infesté par $H$. suaveolens}

La Figure 4 présente les paramètres biophysiques de $H$. suaveolens sous le contrôle des deux provenances de souches de $P$. maximum $\mathrm{C} 1$ après trois mois de culture. Pour tous les paramètres, la performance de production de $H$. suaveolens a été relativement faible en présence de $P$. maximum $\mathrm{C} 1$ provenues d'un milieu préalablement infesté par H. suaveolens. De même, la production de P. maximum $\mathrm{C} 1$ provenant de ce même milieu a été aussi faible comparativement à celle provenant d'un milieu non infesté par $H$. suaveolens. Il y aurait eu un affrontement entre les deux espèces ( $P$. maximum $\mathrm{C} 1$ et $H$. suaveolens qui se seraient reconnues) qui aurait affecté leurs performances de production. Toutefois, les différences observées sous le contrôle des deux provenances de souches de $P$. maximum $\mathrm{C} 1$ entre les paramètres biophysiques de $H$. suaveolens d'une part et ceux de P. maximum $\mathrm{C} 1 \mathrm{~d}$ 'autre part n'ont pas été significatives au seuil de 5\% (Tableau 2). 


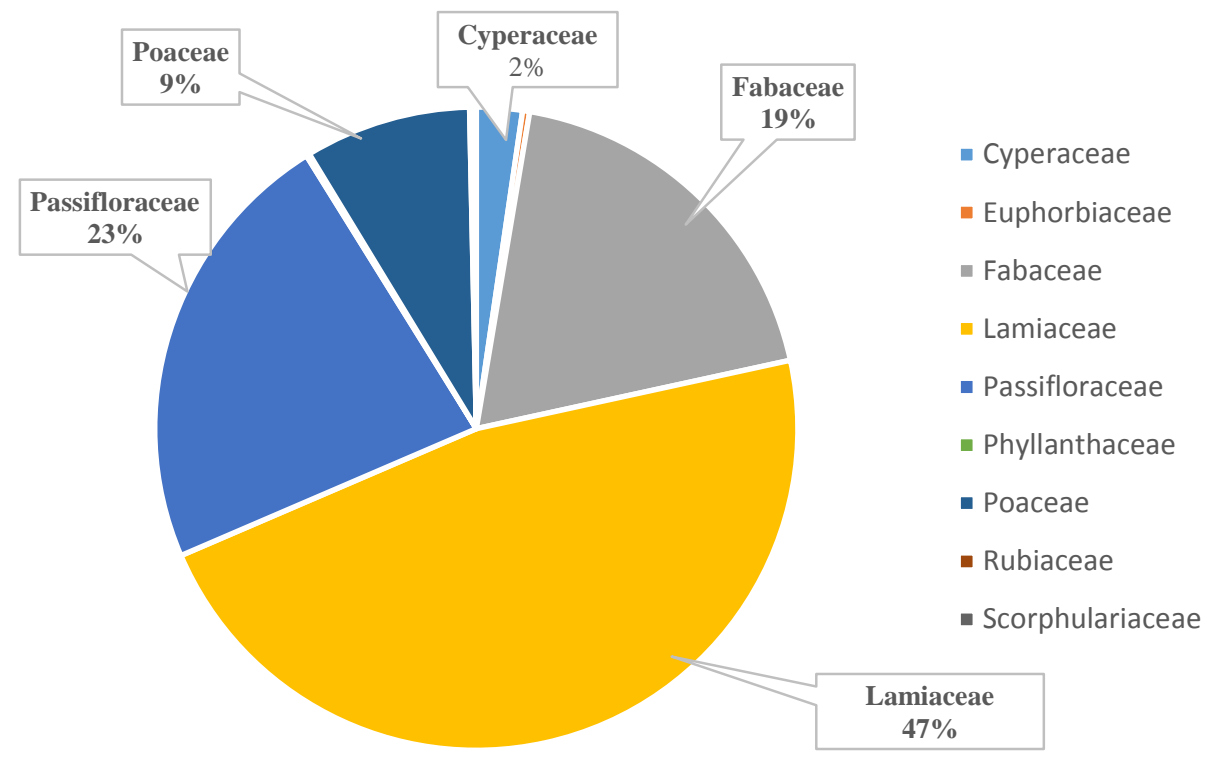

Figure 2 : Contribution spécifique des familles d'espèces dans un pâturage envahi par H. suaveolens.

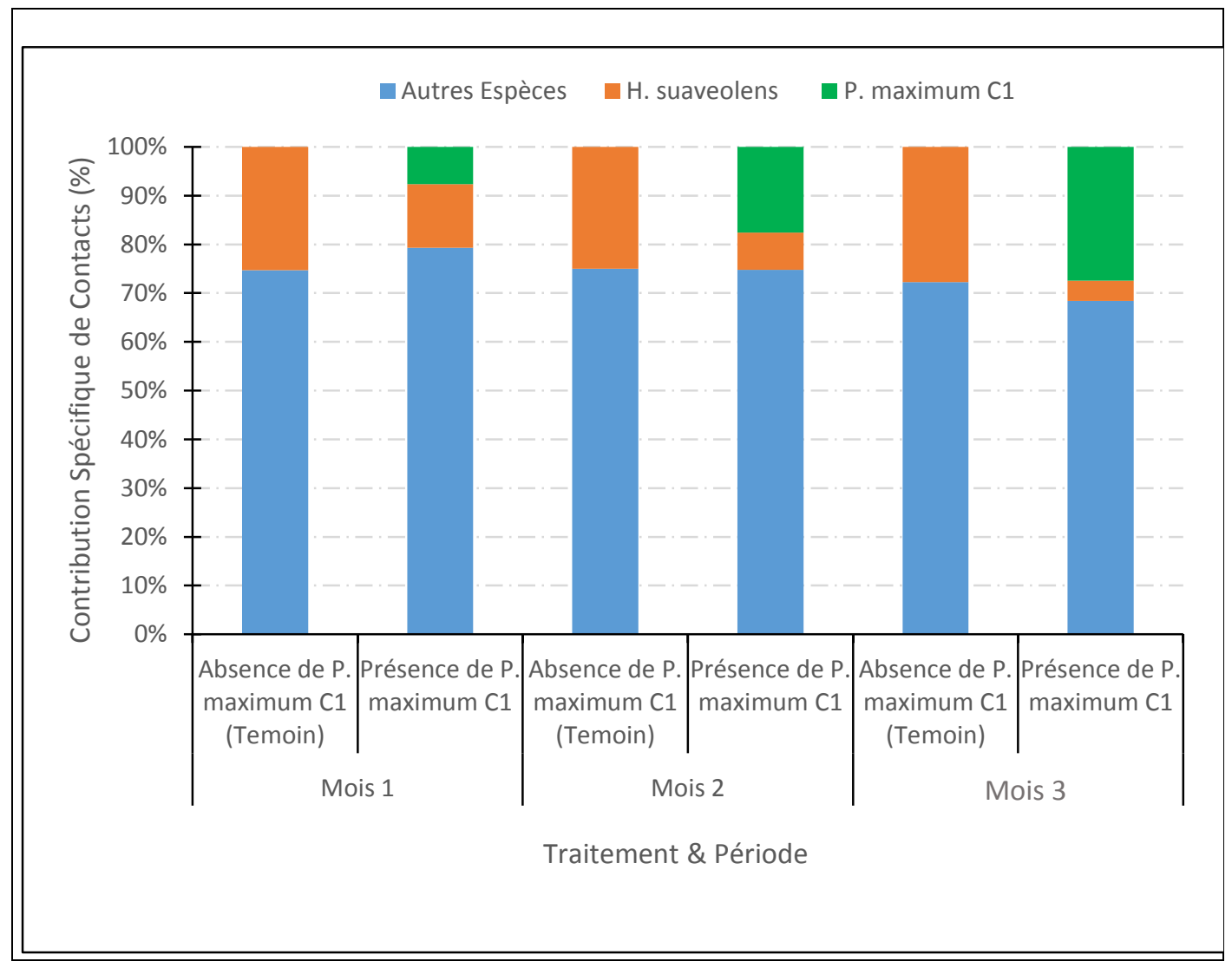

Figure 3: Effet de $P$. maximum $C l$ sur la Contribution Spécifique de Contact (CSC) de $H$. suaveolens et des autres espèces. 
Tableau 1 : Analyse de variance des paramètres biophysiques de catégories d'espèces.

\begin{tabular}{|c|c|c|c|c|c|c|c|c|c|c|}
\hline \multirow[b]{2}{*}{ Paramètre } & \multirow{2}{*}{ Catégorie } & \multicolumn{3}{|c|}{ Mois 1} & \multicolumn{3}{|c|}{ Mois 2} & \multicolumn{3}{|c|}{ Mois 3} \\
\hline & & Df & $F$ value & $\operatorname{Pr}(>\mathbf{F})$ & Df & $F$ value & $\operatorname{Pr}(>\mathbf{F})$ & Df & F value & $\operatorname{Pr}(>\mathbf{F})$ \\
\hline \multirow[t]{3}{*}{$\mathrm{CSC}(\%)$} & P. maximum $\mathrm{C} 1$ & 1 & 6.051 & 0.069 . & 1 & 0.619 & 0.475 & 1 & 0.31 & 0.607 \\
\hline & H. suaveolens & 2 & 7.841 & $0.021 *$ & 2 & 17.46 & $0.003 * *$ & 2 & 32.36 & $<0.001$ \\
\hline & Autres espèces & 2 & 1.12 & 0.384 & 2 & 0.03 & 0.968 & 2 & 0.64 & 0.556 \\
\hline \multirow{2}{*}{$\begin{array}{l}\text { Biovolume } \\
\left(\mathrm{m}^{3} / \mathrm{m}^{2}\right)\end{array}$} & P. тахітит $\mathrm{C} 1$ & 1 & 0.28 & 0.601 & 1 & 0.01 & 0.945 & 1 & 3.57 & 0.0736 \\
\hline & H. suaveolens & 2 & 12.38 & $0.001 * * *$ & 2 & 13.81 & $<0.001$ & 2 & 13.307 & $<0.001$ \\
\hline \multirow{3}{*}{$\begin{array}{l}\text { Biomasse } \\
\text { (KgMS/ha) }\end{array}$} & P. maximum $\mathrm{C} 1$ & & & & & & & 1 & 0.02 & 0.896 \\
\hline & H. suaveolens & & & & & & & 2 & 13.39 & $0.006^{* * *}$ \\
\hline & Autres espèces & & & & & & & 2 & 1.61 & 0.275 \\
\hline
\end{tabular}

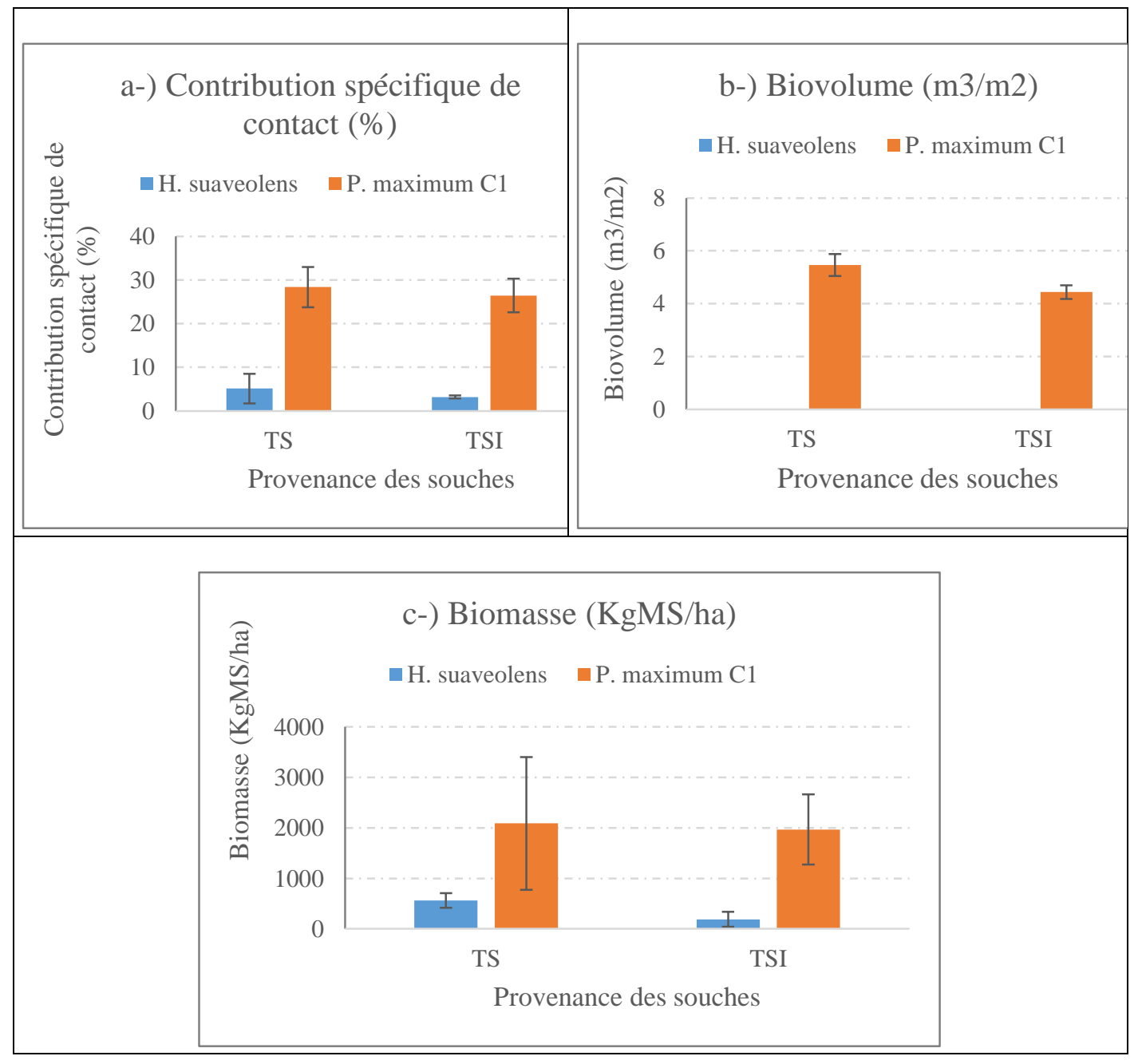

Figure 4 : Effet de provenance de P. maximum $\mathrm{C} 1$ sur le contrôle d'invasion de H. suaveolens. 
Tableau 2 : Comparaison des paramètres biophysiques de $H$. suaveolens et de $P$. maximum $\mathrm{C} 1$ selon le test de Newman-Keuls.

\begin{tabular}{|c|c|c|c|c|c|}
\hline Paramètre & Catégorie & Facteurs & Mois 1 & Mois 2 & Mois 3 \\
\hline \multirow[t]{5}{*}{$\operatorname{CSC}(\%)$} & $\begin{array}{l}P . \quad \text { maximum } \\
\mathrm{C} 1\end{array}$ & TS & $9.98 \pm 3.38 \mathrm{a}$ & $18.75 \pm 4.62 \mathrm{a}$ & $28.37 \pm 4.62 \mathrm{a}$ \\
\hline & & TSI & $5.11 \pm 0.56 \mathrm{a}$ & $16.35 \pm 2.51 \mathrm{a}$ & $26.44 \pm 3.84 \mathrm{a}$ \\
\hline & H. suaveolens & To & $25.05 \pm 2.85 \mathrm{a}$ & $25.01 \pm 6.81 \mathrm{a}$ & $27.64 \pm 6.30 \mathrm{a}$ \\
\hline & & TS & $15.62 \pm 6.92 b$ & $9.39 \pm 2.54 b$ & $5.15 \pm 3.38 \mathrm{~b}$ \\
\hline & & TSI & $10.24 \pm 2.88 \mathrm{~b}$ & $5.85 \pm 0.85 b$ & $3.19 \pm 0.35 b$ \\
\hline \multirow[t]{5}{*}{$\begin{array}{l}\text { Biovolume } \\
\left(\mathrm{m}^{3} / \mathrm{m}^{2}\right)\end{array}$} & $\begin{array}{l}P . \quad \text { maximum } \\
\mathrm{C} 1\end{array}$ & TS & $0.46 \pm 0.21 \mathrm{a}$ & $2.18 \pm 0.81 \mathrm{a}$ & $4.40 \pm 1.01$ \\
\hline & & TSI & $0.42 \pm 0.16 \mathrm{a}$ & $2.16 \pm 0.91 \mathrm{a}$ & $5.46 \pm 1.60$ \\
\hline & H. suaveolens & $\mathrm{T} 0$ & $0.11 \pm 0.071 \mathrm{a}$ & $0.17 \pm 0.13 \mathrm{a}$ & $0.274 \pm 0.255 \mathrm{a}$ \\
\hline & & TS & $0.04 \pm 0.038 b$ & $0.04 \pm 0.04 \mathrm{~b}$ & $0.011 \pm 0.021 \mathrm{~b}$ \\
\hline & & TSI & $0.02 \pm 0.009 \mathrm{~b}$ & $0.01 \pm 0.01 \mathrm{~b}$ & $0.004 \pm 0.001 \mathrm{~b}$ \\
\hline \multirow[t]{5}{*}{$\begin{array}{l}\text { Biomasse } \\
\text { (KgMS/ha) }\end{array}$} & $\begin{array}{l}P . \quad \text { maximum } \\
\mathrm{C} 1\end{array}$ & TS & & & $2086.71 \pm 1313.86 a$ \\
\hline & & TSI & & & $1966.80 \pm 695.51 \mathrm{a}$ \\
\hline & H. suaveolens & To & & & $3185.40 \pm 1322.41 \mathrm{a}$ \\
\hline & & TS & & & $560.92 \pm 146.02 \mathrm{~b}$ \\
\hline & & TSI & & & $189.27 \pm 148.09 \mathrm{~b}$ \\
\hline
\end{tabular}

Les valeurs des facteurs (To, TS, TSI) du même mois, suivies de la même lettre (en colonne) pour le même paramètre ne sont pas significativement différentes au seuil de $5 \%$.

\section{DISCUSSION}

Le cortège floristique du pâturage avant l'installation de l'essai a montré une abondance de Hyptis suaveolens d'environ $47 \%$. Ce qui indique que le processus d'envahissement a atteint le stade 3 d'invasion dans la région (Chabrerie et al., 2006). Ainsi, après le défrichage, de nouvelles populations n'ont pas tardé à se remettre en place conformément aux constats de Radosevich et al. (2003). L'introduction de $P$. maximum $\mathrm{C} 1$ dans le pâturage envahi par $H$. suaveolens a ainsi entraîné une dynamique dans l'expansion de $H$. suaveolens. En effet, au fur et à mesure que $P$. maximum $\mathrm{C} 1$ se développe, $H$. suaveolens devient de moins en moins fréquente, mais constante dans son abondance sur les parcelles témoin. L'introduction de $P$. maximum $\mathrm{C} 1$ a permis d'améliorer fortement la contribution spécifique des graminées et de modifier la composition floristique en faveur de l'alimentation du bétail (Savadogo, 2002).

Par ailleurs, l'abondance de $H$. suaveolens au niveau de la parcelle témoin s'expliquerait par son comportement vis-à-vis des autres espèces. En effet, elle crée de l'ombrage aux autres plantes sous-jacentes à travers son feuillage et limite ainsi le processus photosynthétique chez ces plantes qui jaunissent et meurent. L'ombrage devient plus accentué lorsque $H$. suaveolens atteint le stade $3 \mathrm{~d}$ 'invasion après trois mois ; ce qui justifierait la faible diversité floristique au niveau de la situation initiale avant l'installation de l'essai et sur les parcelles témoins. Ces espèces dont la plupart sont de la famille des Fabaceae, des 
Rubiaceae et des Passifloraceae, sont toutes des annuelles et ne peuvent résister à l'invasion de H. suaveolens (Brondeau et Hivert, 2003; Ngobo et al., 2004 ; Aboh, 2008). Par ailleurs, l'effet allélopathique de $H$. suaveolens sur les autres espèces végétales est peu documenté et mérite d'être élucidé.

Par ailleurs, les résultats ont montré que les autres espèces ont été moins sensibles à la présence de $P$. maximum $\mathrm{C} 1$. Il s'est agi des espèces telles : D. horizontalis, A. ovalifoliusI, I. hirsuta, P. foetida, M. hirtus, R. brasiliensis, $O$. herbaceae et $O$. corymbosa qui résistent temporairement à l'étouffement de $P$. maximum $\mathrm{C} 1$ et $H$. suaveolens qui ont présenté de forte contribution spécifique de contacts même en présence de $P$. maximum $\mathrm{C} 1$. Cependant, Aboh (2008) a montré que le tallage et le recouvrement du sol plus rapide de $P$. maximum $\mathrm{C} 1$ lui offrent une grande capacité à contrôler $H$. suaveolens et autres adventices en présence. La forte contribution spécifique de contacts de ces autres espèces en présence de $P$. maximum $\mathrm{C} 1$ serait due à la faible densité de 4 plants $/ \mathrm{m}^{2}$ comparativement à celles appliquées par Aboh (2008).

Le développement de $P$. maximum $\mathrm{C} 1$ en termes de biovolume est caractéristique de la qualité de la valeur pastorale de parcours amélioré. A l'opposé des parcelles ayants bénéficié de $P$. maximum $\mathrm{C} 1$, les parcelles témoins constituent de pâturage de faible valeur pastorale compte tenu de leur forte contribution spécifique de contact de $H$. suaveolens et de contribution spécifique des bonnes graminées quasiment nulle (Oumorou et al., 2010 ; Aboh et al., 2012a). En effet, la valeur pastorale est proportionnelle à la contribution spécifique des bonnes et moyennes graminées (Houinato, 2001). La culture de $P$. maximum $\mathrm{C} 1$ dans un pâturage envahis par $H$. suaveolens permet d'améliorer la contribution des graminées en général et celle des bonnes graminées en particulier.

La biomasse de bonnes graminées (TS=2087 $\mathrm{kgMS} / \mathrm{ha}$ et TSI=1966 $\mathrm{kgMS} / \mathrm{ha}$ ) obtenue sur le pâturage amélioré par $P$. maximum $\mathrm{C} 1$, en substitut de la biomasse de $H$. suaveolens (3185 $\mathrm{kgMS} / \mathrm{ha})$ sur les parcelles témoins, constitue une solution salutaire pour l'élevage des ruminants dans le contexte actuel d'invasion généralisée des pâturages naturels au Bénin. Ainsi, la lutte contre $H$. suaveolens dans les pâturages naturels pourra connaître de succès à travers l'introduction de $P$. maximum C1 qui améliore sa productivité et sa valeur pastorale et assure la réduction de taux d'embrouillement. En effet, l'introduction généralisée de $P$. maximum $\mathrm{C} 1$ dans les pâturages naturels envahis par $H$. suaveolens pourrait aider à relever à long terme le grand défi de l'élevage des ruminants au Bénin qui se trouve être le contrôle de l'invasion des plantes exotiques. En effet, compte tenu du coût monétaire et de temps pour pratiquer l'arrachage, le sarclage et l'épandage des produits chimiques qui sont peu efficaces et difficiles (Turpie and Heydenrych, 2000 ; D'Antonio and Meyerson, 2002), l'introduction de $P$. maximum $\mathrm{C} 1$ qui est une forme de lutte biologique constituait une solution à long terme, moins coûteuse et respectueuse de l'environnement. En effet, les auteurs avaient bien suggéré l'utilisation des espèces compétitives notamment les graminées pérennes pour contrôler les herbacées envahissantes (D'Antonio and Meyerson, 2002).

En comparant les paramètres biophysiques de $H$. suaveolens et ceux de $P$. maximum $\mathrm{C} 1$ sous le contrôle des deux provenances de souches de $P$. maximum $\mathrm{C} 1$, il a été noté une baisse relative de performance de production de $H$. suaveolens et de $P$. maximum $\mathrm{C} 1$ sur les parcelles implantées avec $P$. maximum $\mathrm{C} 1$ provenues d'un milieu préalablement infesté par $H$. suaveolens. Quoique, la différence observée entre les deux provenances ne soit pas significative au seuil de 5\%, il n'en demeure pas moins vrai qu'il y a eu une réaction liée à leur histoire. En effet, il aurait eu une réaction naturelle de revanche entre $P$. maximum $\mathrm{C} 1$ et $H$. suaveolens qui n'aurait pas été favorable, ni pour l'un, ni pour l'autre, pour la bonne expression de leurs performances de production. Ces résultats relancent encore d'autres réflexions sur l'assertion établie dans le domaine de contrôle de l'invasion qui jusque-là ont montré que les plantes d'une espèce compétitive ayant survécu 
dans un milieu infesté par une plante envahissante sont plus performantes que celles issues d'un milieu non infesté (Ferrero-Serrano et al., 2011 ; Sebade et al., 2012). Dans le cas de la lutte contre $H$. suaveolens dans les pâturages naturels, les souches de $P$. maximum $\mathrm{C} 1$ qui sont introduites, y sont pour de longues années d'exploitation par les ruminants et serviront pour une permanente lutte contre $H$. suaveolens. Ainsi, seul un mode de gestion rationnel des parcours naturels qui implique la mise au repos des parcelles exploitées pourront garantir un meilleur contrôle d'invasion de $H$. suaveolens. Dans tous les cas, l'introduction de $P$. maximum $\mathrm{C} 1$ dans les pâturages naturels envahis par $H$. suaveolens devra apporter un regain de productivité graminéenne de bonne qualité et très appétée par les ruminants.

\section{Conclusion}

L'étude a révélé que la culture de $P$. maximum $\mathrm{C} 1$ à une densité de 4 plants $/ \mathrm{m}^{2}$ permet d'accroître la contribution spécifique des bonnes graminées et la biomasse consommable des pâturages envahis par $H$. suaveolens. Ainsi, son introduction assure l'amélioration de la valeur pastorale et de la productivité des pâturages envahis par $H$. suaveolens. Somme toute, la culture de $P$. maximum $\mathrm{C} 1$ dans les exploitations infestées par $H$. suaveolens est un moyen efficace d'amélioration de ces pâturages. Cette amélioration est une étape dans le processus de contrôle de l'invasion de $H$. suaveolens. La culture de $P$. maximum $\mathrm{C} 1$ peut donc être promue en milieu paysan pour améliorer les pâturages naturels et limiter l'invasion de $H$. suaveolens.

\section{CONFLIT D'INTERETS}

Les auteurs déclarent n'avoir aucun conflit d'intérêts.

\section{CONTRIBUTIONS DES AUTEURS}

SYI est l'étudiant qui a rédigé le protocole de recherche, a participé à la collecte des données de cet article et a rédigé son mémoire de fin de formation de Master; ASY est le coordonnateur du projet CIPAM et a rédigé le manuscrit de cet article, PL a contribué à la révision du protocole de l'étudiant et à la correction du manuscrit, ABA est membre du projet CIPAM, a contribué à la rédaction dudit projet et a contribué à la révision de manuscrit, MRBH a contribué à l'encadrement de l'étudiant et à la révision de son protocole et de son mémoire.

\section{REMERCIEMENTS}

Cette étude a été réalisée dans le cadre du CIPAM intitulé «Contrôle de l'invasion de Hyptis suaveolens Poit dans les pâturages naturels par la culture de Panicum maximum Cl dans les exploitations peulh au Bénin» financé par le Fonds National de la Recherche Scientifique et de l'Innovation Technologique (FNRSIT). Les auteurs remercient l'Institut National des Recherches Agricoles du Bénin (INRAB) et la Faculté des sciences Agronomiques de l'Université d'AbomeyCalavi pour les facilités accordées pour la bonne exécution dudit projet.

\section{REFERENCES}

Aboh BA. 2008. Phytosociologie, écologie, potentialités et aménagement des pâturages naturels envahis par Chromolaena odorata et Hyptis suaveolens en Zone Soudano-guinéenne (Bénin). Thèse de Doctorat, Université d'Abomey-Calavi, Bénin, 227 p.

Aboh BA, Oumorou M, Houinato M, Sinsin B. 2009. Analyse biologique et phytogéographique des savanes colonisées par Chromolaena odorata et Hyptis suaveolens dans la région de Bétécoucou (Bénin). Systematics and Geography of Plant, 79: 81-92. DOI: https://www.jstor.org/stable/20649773

Aboh BA, Babatounde S, Oumorou M, Houinato M, Sinsin B. 2012a. Valeur pastorale des parcours naturels en zone soudano-guinéenne et stratégie paysanne d'adaptation aux effets de leur invasion par Chromolaena odorata au Bénin. International Journal of Biological and Chemical Sciences, 6(4): 1633-1646. DOI: http://dx.doi.org/10.4314/ijbcs.v6i4.22. 
Aboh BA, Zoffoun AG, Djenontin JA, Babatounde S, Mensah GA. 2012b. Fiche technique : Strategie d'adaptation de la charge animale à la production des parcours naturels envahis par Hyptis suaveolens. Bibliothèque nationale $\mathrm{du}$ Bénin, Cotonou, p. 6.

Brondeau A, Hivert J. 2003. Plantes exotiques envahissantes : Méthode de lutte mises en œuvre par l'Office National des Forêts de la Réunion. In Proceedings of the regional workshop on invasive alien species and terrestrial ecosystem rehabilitation in western Indian Ocean islands states; 3442.

CABI (Centre for Agriculture and Biosciences International). 2004. Prevention and management of alien invasive species. Forging Cooperation throughout West Africa, CABI-Workshop held Accra: Ghana; 9-11 march.

http://www.gisp.org/downloadpubs/West Africa.pdf.

César J. 2005. La culture fourragère à base de Panicum maximum. Fiche $\mathrm{N}^{\circ} 15$, p. 8.

Chabrerie O, Hoeblich H, Decocq G. 2006. Déterminisme et conséquences écologiques de la dynamique invasive du cerisier tardif (Prunus serotina Ehrh.) sur les communautés végétales de la forêt de Compiègne. Acta Botanica Gallica, 153: 383-394.

DOI: https://login.research4life.org/tacsgr1doi _org/10.1080/12538078.2007.10516071.

CRA Nord. 2018. INRAB/MAEP, Rapport Technique et Financier du $1^{\text {er }}$ janvier au 31 décembre 2018 du Centre de Recherches Agricoles Nord basé à Ina, 41 p.

D'Antonio C, Meyerson LA. 2002. Exotic plant species as problems and solutions in ecological restoration: a synthesis. Restoration Ecology, 10(4): 703-713. DOI: https://doi.org/10.1046/j.1526100X.2002.01051.x.

Daget P, Poissonet J. 1971. Une méthode d'analyse phytologique des prairies: Critères d'application. Annales Agronomiques, 22: 5-41. DOI: https://agritrop.cirad.fr/537178/
Faure P. 1977. Notice explicative de la carte pédologique de reconnaissance de la R.P. Bénin. ORSTOM Paris, 49 p.

Ferrero-Serrano A, Hild AL, Mealor BA. 2011. Can invasive species enhance competitive ability and restoration potential in native grass populations? Restoration Ecology, 19: $545-551 . \quad$ DOI: https://doi.org/10.1111/j.1526100X.2009.00611.x.

Houinato M. 2001. Phytosociologie, production et capacité de charge des formations végétales pâturées dans la région des Monts Kouffé (Bénin). Thèse de Doctorat, Université Libre de Bruxelles, Belgique, $219 \mathrm{p}$.

Holou RAY, Achigan-Dako EG, Sinsin B. 2013. Ecology and management of invasive plants in Africa In Invasive Plant Ecology, Shibu J, Harminder PS, Daizy RB, Ravinder KK (Eds). CRC Press: USA; 161-174.

Lesse P. 2016. Gestion et modélisation de la dynamique des parcours de transhumance dans un contexte de variabilites climatiques au Nord-Est du Bénin. Thèse de Doctorat, Université d'AbomeyCalavi, Bénin, 299 p.

Messager JL. 1984. Note sur les modalités techniques d'installation des pâturages de Panicum. Revue de l'élevage et Médecine Vétérinaire des Pays Tropicaux, 37(3): 336-340.

DOI: https://doi.org/10.19182/remvt.8421

Ngobo M, Mcdonald M, Weise S. 2004. Impacts of type of fallow and invasion by Chromolaena odorata on weed communities in crop fields in Cameroon. Ecology and Society, 9(2): 1. DOI: http://www.ecologyandsociety.org/vol9/i ss $2 / \operatorname{art} 1 /$

Oumorou M, Aboh BA, Babatounde S, Houinato M, Sinsin B. 2010. Valeur pastorale, productivité et connaissances endogènes de l'effet de l'invasion, par Hyptis suaveolens L. Poit., des pâturages naturels en zone soudano-guinéenne (Bénin). Int. J. Biol. Chem. Sci., 4(4): 1262-1277.

DOI: https://doi.org/10.4314/ijbcs.v4i4.63062 
Radosevich SR, Stubbs MM, Ghersa CM. 2003. Plant invasions process and patterns. Weed Science, 5(1): 254-259. DOI: $\quad$ https://doi.org/10.1614/00431745(2003)051[0254:PIPAP]2.0.CO;2.

Savadogo P. 2002. Pâturages de la forêt classée de Tiogo: Diversité végétale, productivité, valeur nutritive et utilisations. Mémoire de fin d'étude, IDR/UPB, $105 \mathrm{p}$.

Sebade BM, Hild AL, Mealor BA. 2012. Native grasses collected from invasions demonstrate invasion resistance. Ecological Restoration, 30: 209-217. DOI: 10.3368/er.30.3.209.

Sinsin B. 1993. Phytosociologie, écologie, valeur pastorale, productivité et capacité de charge des pâturages naturels du périmètre Nikki-Kalalé au Nord Bénin. Thèse de doctorat, Université Libre de Bruxelles, Belgique, p. 390.

Thiombiano DNE. 2008. Etude de trois espèces à tendance prolifique et envahissante dans la province du Boulkiemdé : Essai de valorisation (Burkina Faso). Mémoire d'Ingénieur des Eaux et Forêts, IDR/UPB, $126 \mathrm{p}$.

Thiombiano N, Ouedraogo RL, Belem M, Guinko S. 2009. Dynamique de l'évolution et impact d'une plante envahissante au Burkina Faso : Hyptis suaveolens (L.) Poit. Annales de l'Université de Lomé (Togo), XVIII : 97115.

Turpie J, Heydenrych B. 2000. Economic consequences of alien infestation of the Cape Floral Kingdom's Fynbos vegetation. In The Economics of Biological Invasions, Perrings C, Williamson M, Dalmazzone S (eds). Elgar: Cheltenham; 214-261.

Youssouf I. 1990. Etude pédologique au 1/10000 du périmètre de SRCV-Ina. Bibliothèque, DRA Cotonou et Ina, 87 p.

Willaine P. 1965. Notice explicative de la carte pédologique au 1/5000 de la station d'Ina. Centre ORSTOM de Cotonou, Benin, 16 p. 